\title{
Pediatric pacemaker infections: Twenty years of experience
}

\author{
Mitchell I. Cohen, MD \\ David M. Bush, MD, PhD ${ }^{a}$ \\ J. William Gaynor, MD $^{\mathrm{b}}$ \\ Victoria L. Vetter, MD ${ }^{\mathrm{a}}$ \\ Ronn E. Tanel, MD ${ }^{\mathrm{a}}$ \\ Larry A. Rhodes, MD
}

From the Divisions of Cardiology ${ }^{\mathrm{a}}$ and Cardiothoracic Surgery ${ }^{\mathrm{b}}$ and the Departments of Pediatrics and Surgery, The Children's Hospital of Philadelphia and The University of Pennsylvania School of Medicine, Philadelphia, Pa.

Presented in part at the 74th Annual Meeting of the American Heart Association, Anaheim, Calif, November 14, 2001.

Received for publication Nov 2, 2001; revisions requested Dec 13, 2001; revisions received Jan 8, 2002; accepted for publication Feb 10, 2002.

Address for reprints: Mitchell I. Cohen, MD, Division of Pediatric Cardiology, The Children's Hospital of Philadelphia, 34th \& Civic Center Blvd, Philadelphia, PA 19104 (E-mail: cohenmi@email.chop.edu).

J Thorac Cardiovasc Surg 2002; 124:821-7

Copyright (ㄷ 2002 by The American Association for Thoracic Surgery

$0022-5223 / 2002 \$ 35.00+0 \quad \mathbf{1 2 / 1 / 1 2 3 6 4 3}$

doi: $10.1067 / \mathrm{mtc} .2002 .123643$

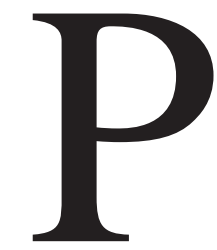

Objective: We sought to evaluate possible predictors of early and late pacemaker infections in children.

Methods: A review was performed of all pacemakers implanted in children at The Children's Hospital of Philadelphia between 1982 and 2001. Infections were classified as superficial cellulitus, deep pacemaker pocket infection necessitating removal, or positive blood culture without an identifiable source.

Results: A total of 385 pacemakers (224 epicardial and 161 endocardial) were implanted in 267 patients at $8.4 \pm 6.2$ years. All 2141 outpatient visits were reviewed (median follow-up, 29.4 months; range, 2-232 months). There were 30 (7.8\%) pacemaker infections: 19 (4.9\%) superficial infections; 9 (2.3\%) pocket infections; and $2(0.5 \%)$ isolated positive blood cultures. All superficial infections resolved with intravenous antibiotics. The median time from implantation to infection was 16 days (range, 2 days-5 years). Only 1 deep infection occurred after primary pacemaker implantation. Six patients with deep infections were pacemaker dependent and were successfully managed with intravenous antibiotics, followed by lead-generator removal and implantation of a new pacemaker in a remote location. In univariate analyses trisomy 21 (relative risk, $3.9 ; P<$ .01 ), pacemaker revisions (relative risk, $2.5 ; P<.01$ ), and single-chamber devices (relative risk, 2.4; $P<.05$ ) were identified as predictors of infection. However, in multivariate analyses only trisomy 21 and pacemaker revisions were predictors.

Conclusions: The incidences of superficial and deep pacemaker infections were $4.9 \%$ and $2.3 \%$, respectively. Trisomy 21 and pacemaker revisions were significant risk factors in the development of infection after pacemaker implantation. For primary pacemaker implantation, the risk of infection requiring system removal is low $(0.3 \%)$. infections have been reported in $1 \%$ to $2 \%$ of adult patients and often require removal of the infected generator and lead or leads. ${ }^{3-5}$ However, little information currently exists concerning early and late pacemaker-related infections in children. This study was undertaken to assess the incidence and possible 


\section{TABLE 1. Variables entered into univariate analyses}

Age at implantation

Weight at implantation

Cardiac malformation

Era of implantation (1980-85, 1985-1990, 1990-1995, 1995-2001)

Trisomy 21

Cardiac surgical procedures (type and number)

Surgical approach (transvenous vs epicardial)

Concurrent cardiac procedure

Indication for pacing

Chamber paced

Single- vs dual-chamber pacemaker

Lead fixation

Initial implantation vs pacemaker revision

predictors of pediatric pacemaker infections in a large single-center cohort over 2 decades.

\section{Methods}

The cardiac surgical and pacemaker databases were reviewed to identify patients who underwent permanent pacemaker implantation between July 1, 1982, and June 30, 2001, at The Children's Hospital of Philadelphia. Patients older than 21 years at the time of pacemaker implantation were excluded. All hospital and operative records, as well as the 2141 outpatient pacemaker clinic visits, were retrospectively reviewed. These records were abstracted for potential predictors of pacemaker infections (Table 1).

\section{Operative Techniques}

Epicardial leads were implanted by using standard surgical techniques either through a midline sternotomy, lateral thoracotomy, or subxiphoid approach. The surgical approach was based on the patient's underlying cardiac anatomy, morphologic cardiac position in situ, previous operations, and/or concurrent operation at the time of lead placement. The ventricular lead was often fixed on the diaphragmatic ventricular surface. The atrial lead was positioned on either the right or left atrium. The surplus of lead was addressed by creating loops of the electrode within the pericardium and the pacemaker pocket. Leads were tunneled with care to avoid sites of potential injury along the rib margins to the generator implant site in the abdomen.

Endocardial leads were implanted through percutaneous puncture of the right or left subclavian vein. In rare instances cutdown of either the subclavian or cephalic vein was performed. By using a standard Seldinger technique, a guide wire was advanced, and a sheath was secured over the guide wire and the pacing lead or leads positioned in the atrium, ventricle, or both. The generator was placed in either a subcutaneous or subpectoral position. Intravenous antibiotics, typically cefazolin, were given intraoperatively and then for 24 to 48 hours after pacemaker implantation.

\section{Hospital Course and Follow-up}

Hospital charts were reviewed for length of stay, complications, number and duration of antibiotics received, and discharge pacing characteristics. Records were also reviewed for all isolated generator changes without lead revisions and subsequent complications. Outpatient pacemaker follow-up generally occurred 1 and 3 months after implantation and then biannually. Perioperative in- fections were defined as occurring before discharge after the initial pacemaker implantation. Early complications were defined as any infection requiring readmission or additional diagnostic examinations (blood culture and wound aspiration) during the first 60 days after pacemaker implantation. Readmission for bacteremia, skin erosion, or pacemaker infection beyond 60 days was considered a late complication. Pacemaker infections were based on modifications to criteria by Choo and associates ${ }^{6}$ and included the following: (1) superficial infection (with or without a positive blood culture); (2) deep infection necessitating generator-lead removal; or (3) fever associated with positive blood culture without a focus of infection elsewhere. All records at readmission were reviewed with specific attention to duration and type of intravenous and oral antibiotics used, wound and blood culture results, and need for removal of the generator, extraction of the lead or leads, or both.

\section{Statistical Analysis}

Exploratory data analysis was performed by using descriptive measures. Categoric variables were expressed as percentages with associated SDs. Continuous variables were expressed as means with SDs if the term was normally distributed, and skewed variables were expressed as medians with ranges. The strength of statistical associations between categoric factors was assessed by using $\chi^{2}$ tests for categoric variables. When the expected cell numbers were small, the Fisher exact test was used. Statistical significance of the difference between continuous variables was assessed with the Wilcoxon rank sum test for skewed distribution. Logistic regression modeling was used to assess potential confounding variables with STATA 6.0 software (College Station, Tex).

\section{Results}

\section{Patient Data}

A total of 267 patients (113 female and 154 male patients) underwent 385 pacemaker implantations during the 20-year study period. Of these 267 patients, $172(64 \%)$ continued to use their primary pacemaker system throughout the study period without either a lead change, generator change, or both. Within the total cohort, 59 (22\%) patients underwent a combination of 76 reoperations for generator and lead replacement. Of the 267 patients, 36 underwent 42 (9\%) elective isolated generator changes. The median age at primary pacemaker implantation was 7.3 years $(<3$ months of age, $\mathrm{n}=36 ; 3$ months- 1 year, $\mathrm{n}=13 ; 1-5$ years, $\mathrm{n}=61$; $5-10$ years, $\mathrm{n}=56$; and $>10$ years, $\mathrm{n}=101)$. The median weight at lead implantation was $24.9 \mathrm{~kg}$ (range, 1.4-156 kg). Indications for pacemaker placement included postoperative atrioventricular block in $83(31 \%)$ patients, sinus node dysfunction in 90 (34\%) patients, congenital complete heart block in 54 (20\%) patients, bradycardia associated with long QT syndrome in 14 (5.3\%) patients, and other indications, such as bradycardia caused by antiarrhythmic medication, ventricular tachycardia, or antitachycardia pacemakers in 26 (9.7\%) patients.

The majority of our patients (70\%) had structurally abnormal hearts and had undergone surgical correction of 
complex cardiac malformations (Table 2). Of the 187 patients with congenital heart disease, 62 (33\%) had some form of single-ventricle physiology. Trisomy 21 was present in $23(8.6 \%)$ patients. Follow-up was complete to June 30, 2001, in $91.9 \%$ of patients for a median of 29.4 months (range, 2-232 months).

\section{Pacing Characteristics}

The initial pacing mode (excluding generator changes) used was atrial in 26 patients, ventricular without atrial synchronization in 154 patients, atrioventricular in a synchronized mode in 130 patients, and atrial sensed and ventricular paced in 17 patients. Sixteen patients underwent implantation of an automatic internal cardioverter defibrillator.

\section{Operative and Perioperative Course}

Epicardial pacing systems accounted for 58\% $(\mathrm{n}=224)$ of generators used, and endocardial systems contributed to the remaining $42 \%(\mathrm{n}=161)$. Epicardial leads were implanted through a subxiphoid approach (26 [13\%]), lateral thoracotomy (41 [21\%]), or a sternotomy (129 [66\%]). Endocardial leads were implanted through a left (111 [75.5\%]) or right (32 [22\%]) subclavian venipuncture, cephalic cutdown (3 [2\%]), or internal jugular approach (1 [0.5\%]). A temporary pacing lead was present at the time of permanent pacemaker implantation in 55 of the 343 lead and generator implants (16\%; epicardial, 45/196; endocardial, 10/147). For the 147 primary endocardial implants, the pacemaker pocket was subpectoral in $82(56 \%)$ systems. In the remaining 65 (44\%) implants, the generator was subcutaneously positioned.

Implantation of the pacemaker occurred concurrently with a cardiac procedure in $37(9.6 \%)$ patients, typically after cardiopulmonary bypass. In 92 (24\%) patients implantation of the generator occurred within 14 days of cardiac surgery, most typically as a result of postoperative atrioventricular block. The remainder of the pacemaker $(n=256$ [66.4\%]) implantations occurred at a time remote from either reparative or palliative cardiac surgery. Chest tubes were implanted in 108 patients for an average of 1 day (range, 0-27 days). Antibiotics (cefazolin, vancomycin, or both) were given to all patients intraoperatively and then for an additional 24 to 48 hours in $98 \%$ of the cohort. Prophylactic antibiotics were not routinely used postoperatively before 1988. The median length of stay after an isolated pacemaker implantation was 3 days (range, 1-122 days).

\section{Perioperative Infections (Before Hospital Discharge)}

Five $(1.2 \%)$ patients has a superficial skin infection before hospital discharge after the initial pacemaker implantation. The median time from implantation to the development of superficial cellulitus was 7 days (range, 2-15 days). In one patient Staphylococcus epidermidis grew from the wound aspirate. In the remaining 4 patients, the wound cultures
TABLE 2. Anatomic features of patients receiving pacemakers $(n=267)$

\begin{tabular}{lr}
\hline Structurally normal heart & 80 \\
Single-ventricle heart disease & 62 \\
Tricuspid atresia, NRGA & 7 \\
Tricuspid atresia, TGA & 19 \\
Hypoplastic left heart syndrome & 18 \\
Single RV & 9 \\
Heterotaxy & 5 \\
Other & 4 \\
Complete common AV canal & 24 \\
TGA (S,D,D) & 18 \\
Ventriculoseptal defect & 15 \\
Tetralogy of Fallot & 12 \\
Complex LVOTO & 11 \\
TGA (S,L,L) (士VSD) & 9 \\
Double-outlet right ventricle & 5 \\
Primum ASD & 5 \\
Secundum ASD & 5 \\
Heterotaxy syndrome and AV canal & 4 \\
Partial anomalous pulmonary venous return & 4 \\
Hypertrophic cardiomyopathy & 2 \\
Patent ductus arteriosus & 2 \\
Mitral valve replacement & 2 \\
Truncus arteriosus & 1 \\
Pulmonary stenosis & 1 \\
Dilated cardiomyopathy & 1 \\
Anomalous left coronary artery & 1 \\
S/P orthotopic heart transplant & 1 \\
Ebstein anomaly & 1 \\
\hline
\end{tabular}

NRGA, Normally related great arteries; TGA, transposition of the great arteries; $R V$, right ventricle; $A V$, atrioventricular; $L V O T O$, left ventricular outflow tract obstruction; $V S D$, ventricular septal defect; $A S D$, atrial septal defect; $S / P$, status post.

revealed no growth. None of these 5 patients had a positive blood culture. All 5 patients were successfully treated with an additional 7-day course of intravenous antibiotics (1 cefazolin, 2 vancomycin, and 1 oxacillin). None of these 5 patients were readmitted at a later date with a recrudescent pacemaker infection.

One $(0.2 \%)$ deep-pocket infection occurred 14 days after implantation of an epicardial atrial pacing lead at the time of resection of fibrous subaortic stenosis in a patient after Fontan surgery. Wound cultures revealed Pseudomonas aeruginosa and a coagulase-positive Staphylococcus species. This patient received 2 weeks of intravenous vancomycin, tobramycin, and ciprofloxacin and was eventually discharged home with oral ciprofloxacin for a period of 3 months. However, within 55 days, the patient was readmitted, with erythema and swelling over the generator site. The patient was started on intravenous tobramycin and ticarcillin and within 72 hours underwent division of the lead with generator removal and placement of a new epicardial ventricular pacemaker through a left lateral thoracotomy. The generator was tunneled to a remote preperitoneal abdominal location. The wound culture from the old generator site grew $P$ aeruginosa, for which the patient received 3 months 
TABLE 3. Clinical and bacteriologic data from the 9 deep pacemaker infections

\begin{tabular}{|c|c|c|c|c|c|c|c|c|c|c|}
\hline $\begin{array}{l}\text { Case } \\
\text { no. }\end{array}$ & $\begin{array}{l}\text { Age/ } \\
\text { sex }\end{array}$ & $\begin{array}{l}\text { Primary } \\
\text { implant }\end{array}$ & $E / T$ & CHD & $\begin{array}{l}\text { Type } \\
\text { of } \\
\text { pacer }\end{array}$ & $\begin{array}{l}\text { Time (days) } \\
\text { implant to } \\
\text { infection }\end{array}$ & Etiology & Treatment & Outcome & $\begin{array}{l}\text { Recurrent } \\
\text { infection }\end{array}$ \\
\hline 1 & $14 / F$ & No & $E$ & $\begin{array}{l}\text { Single ventricle, } \\
\text { Fontan }\end{array}$ & VVI & 12 & $\begin{array}{l}\text { Pseudomonas } \\
\text { aeruginosa }\end{array}$ & $\begin{array}{l}\text { Tobramycin and } \\
\text { ticarcillin } \\
1 \text { mo; } \\
\text { ciprofloxacin } \\
3 \text { mo }\end{array}$ & $\begin{array}{l}\text { Partial lead/generator } \\
\text { removed; changed to } \\
\text { remote site }\end{array}$ & Yes \\
\hline 2 & $.2 / \mathrm{M}$ & Yes & $E$ & HLHS/Norwood & AAI & 8 & Serratia & $\begin{array}{l}\text { Gentamicin and } \\
\text { ceftriaxone }\end{array}$ & $\begin{array}{l}\text { Died (low cardiac output } \\
\text { after Norwood) }\end{array}$ & No \\
\hline 3 & 4/M & No & $E$ & $\begin{array}{l}\text { Tetralogy of } \\
\text { Fallot }\end{array}$ & VVI & $16^{*}$ & 0 & Ceftriaxone $14 \mathrm{~d}$ & $\begin{array}{l}\text { Lead/generator removed } \\
\text { (adequate escape) }\end{array}$ & No \\
\hline 4 & $6 / F$ & No & $E$ & $\begin{array}{l}\text { Single ventricle, } \\
\text { Fontan }\end{array}$ & VVI & 34 & $\begin{array}{l}\text { Pseudomonas } \\
\text { aeruginosa }\end{array}$ & $\begin{array}{l}\text { Vancomycin and } \\
\text { netilmicin } 14 \mathrm{~d}\end{array}$ & $\begin{array}{l}\text { Lead/generator removed; } \\
\text { changed to remote } \\
\text { site }\end{array}$ & No \\
\hline 5 & $10 / \mathrm{M}$ & No & $\mathrm{T}$ & $\begin{array}{l}\text { Trisomy 21, AV } \\
\text { canal }\end{array}$ & VVI & $14^{*}$ & $\begin{array}{r}\text { Staph species } \\
\text { (broth only) }\end{array}$ & Ceftriaxone $14 \mathrm{~d}$ & $\begin{array}{l}\text { Pocket aspirated; } \\
\text { resolved with } \\
\text { antibiotics }\end{array}$ & No \\
\hline 6 & $.7 / \mathrm{M}$ & No & $E$ & $\begin{array}{l}\text { Single ventricle, } \\
\text { Glenn }\end{array}$ & DDD & 35 & Klebsiella & $\begin{array}{l}\text { Vancomycin } 14 \\
\text { d; cephalexin } \\
14 \mathrm{~d}\end{array}$ & $\begin{array}{l}\text { Lead/generator removed; } \\
\text { changed to remote site }\end{array}$ & No \\
\hline 7 & $17 / \mathrm{M}$ & No & $\mathrm{T}$ & None & DDD & 176 & $\begin{array}{r}\text { Staph species } \\
\text { (broth only) }\end{array}$ & $\begin{array}{l}\text { Vancomycin and } \\
\text { ancef } 5 \mathrm{~d} \text {; } \\
\text { Aug } 14 \mathrm{~d}\end{array}$ & $\begin{array}{l}\text { Lead/generator removed; } \\
\text { changed to epicardial }\end{array}$ & No \\
\hline 8 & $10 / F$ & No & $E$ & $\begin{array}{l}\text { Trisomy 21, AV } \\
\text { canal }\end{array}$ & VVI & 301 & Staph aureus & $\begin{array}{l}\text { Vancomycin and } \\
\text { gentamicin } \\
10 \mathrm{~d} ; \\
\text { ciprofloxacin } \\
14 \mathrm{~d}\end{array}$ & $\begin{array}{l}\text { Partial lead/generator } \\
\text { removed; changed to } \\
\text { remote site }\end{array}$ & Yes \\
\hline 9 & $11 / \mathrm{M}$ & No & $E$ & Aortic stenosis & VVI & 1736 & 0 & $\begin{array}{l}\text { Vancomycin } 7 \mathrm{~d} ; \\
\text { aug } 14 \mathrm{~d}\end{array}$ & $\begin{array}{l}\text { Lead/generator removed; } \\
\text { changed to remote site }\end{array}$ & No \\
\hline
\end{tabular}

$E$, Epicardial; $T$, transvenous; $H L H S$, hypoplastic left heart syndrome; $A V$, atrioventricular.

${ }^{*}$ Generator change only.

of intravenous antibiotics and an additional 6 months of oral ciprofloxacin. The old abdominal generator site was irrigated, packed daily, and closed by secondary intent. No complications ensued from the subsequently placed ventricular generator. However, the patient returned 10 months later with erythema around the original pacemaker pocket. A computed tomographic scan revealed fluid collection around the previously transected ventricular lead. An incision extending into the subxiphoid region identified the old epicardial lead, which was completely removed from the diaphragmatic surface of the right ventricle. The patient recovered without further complications.

\section{Early Pacemaker Infections (<60 Days After Implantation)}

Twelve $(3.1 \%)$ patients had a superficial wound infection within 60 days of pacemaker implantation (18 days; range, 2-37 days). Of these 12 patients, 11 occurred after implantation of a primary pacing system, whereas one superficial infection occurred solely after an elective generator change. In all 12 patients, a peripheral blood culture before initiation of antibiotics revealed no organism. A wound aspirate from one individual grew Staphylococcus aureus, and all other wound cultures were negative. Patients were managed with intravenous cefazolin for 2 to 7 days and then with an additional 7 to 14 days of oral cephalexin on an outpatient basis. No patient required removal of the lead, generator, or both. No patient required readmission for a worsening infection requiring additional antibiotics. In all 12 patients, a follow-up outpatient visit 2 weeks after discharge revealed resolution of the superficial erythema.

An early deep pacemaker pocket infection was observed in 5 (1.2\%) patients (implant: median age, 4.1 years; range, 2 weeks-10 years; weight at implant: $16.3 \mathrm{~kg}$; range, $3-28 \mathrm{~kg}$ ) (Table 3). Of these 5 patients, 2 had a deep-pocket infection after a lead revision and generator change, and 2 others occurred after an elective generator change. The median time from pacemaker implantation to deep-pocket infection was 16 days (range, 8-47 days). All 5 patients had complex congenital heart disease, and 3 had single-ventricle physiology. In 4 of the 5 patients, the pacemaker revision occurred without an asso- 
ciated nonpacemaker cardiac procedure. In one patient with hypoplastic left heart syndrome, the primary pacing system was implanted 3 weeks after a stage I Norwood procedure for sinus bradycardia and low cardiac output. This patient eventually had Serratia marcesans septicemia and required removal of the generator; the patient died 3 weeks later.

In the remaining 4 patients with an early deep-pocket infection, microbiologic studies revealed no organism in one patient, Klebsiella species (wound and blood) in one patient, $P$ aeruginosa (wound) in one patient, and an unidentified Staphylococcus species (blood) in one patient. All 4 patients had removal of the generator, and 3 also underwent lead extraction. A new pacemaker system was implanted in 2 patients after a brief period of intravenous antibiotics (7-10 days) at a remote site. One patient with trisomy 21 and complete heart block after atrioventricular canal repair underwent exteriorization of the generator, 2 weeks of intravenous ceftriaxone, and daily dressing changes. A new generator connected to the original epicardial leads was successfully implanted without reinfection. One patient with complete heart block after repair of tetralogy of Fallot had been recently given a diagnosis of stage IV neuroblastoma and underwent an elective generator change for endof-life battery indices. The patient had a deep-pocket infection during a period of severe neutropenia while receiving chemotherapy. As a result of having an adequate escape rhythm (ventricular rate, 70 beats/min), the family and physicians elected to refrain from pacemaker reimplantation until the patient recovered from neuroblastoma.

One 2-month-old patient with third-degree atrioventricular block after repair of a ventricular septal defect had isolated Enterococcus bacteremia and received 42 days of ampicillin and gentamicin. The patient recovered without incident and did not require the generator, lead, or both to be removed.

\section{Late Pacemaker Infections (>60 Days After Implantation)}

A late superficial pacemaker infection was identified in 2 (0.5\%) patients (aged 2 and 11 years) 136 and 211 days, respectively, after an isolated implantation of a singlechamber ventricular epicardial pacing system. Blood cultures were negative in both patients, and each responded to a brief ( $<5$ days) course of intravenous antibiotics and an additional 7 days of oral antibiotics. Neither late superficial infection progressed to a deep-pocket infection necessitating pacemaker removal.

Two pacemaker-dependent patients with epicardial leads and one patient with an endocardial system had a late deep-pocket infection necessitating pacemaker removal. A gram-positive species was identified in the wound aspirate of 2 patients; however, both had negative blood cultures. One of these patients, a 10-year-old girl with trisomy 21, sustained an epicardial lead fracture and underwent place-

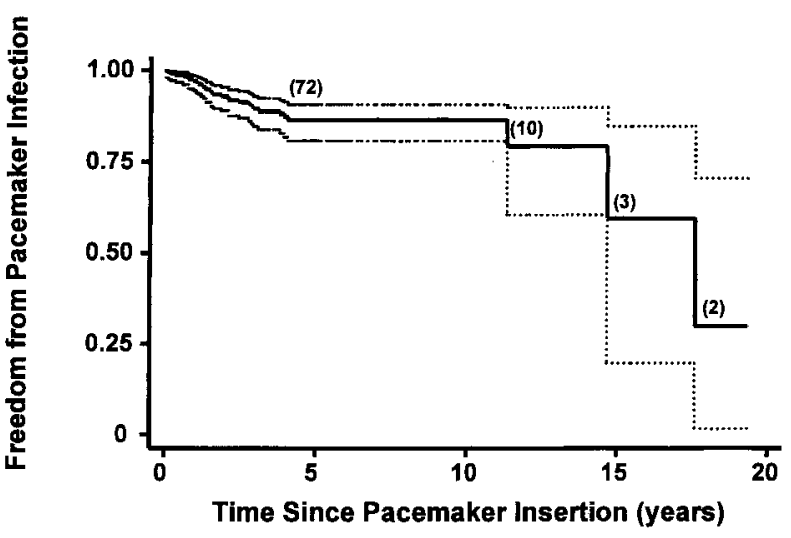

Figure 1. Freedom from pacemaker infection. Dashed lines denote 95\% confidence limits about the point estimates. Numbers in parentheses denote number of patients at that time point for whom follow-up data were available. The 1-, 2-, 5-, and 10-year percentages of freedom from pacemaker infection (with confidence limits) are 96.8 (93.9-98.3), 92.9 (88.9-95.5), 86.5 (80.7-90.7), and 86.5 (80.7-90.7). Three individuals had pacemaker infections after 10 years.

ment of a new epicardial ventricular pacing system. The patient did well initially and 9 months later underwent abdominal exploration for a possible ventral hernia, which revealed infection at the previous fractured pacing lead. The generator was removed, and the fractured epicardial lead was cut and allowed to retract. Despite 1 week of intravenous antibiotics, the patient presented with a purulent subxiphoid fluid collection ( $S$ aureus) requiring reopening of the lower portion of the sternum and removal of the previously cut epicardial lead. A new transvenous system was placed with a subpectoral pocket. In all 3 pacemakerdependent patients, 2 to 5 days of intravenous antibiotics preceded generator and total lead extraction, with concomitant implantation of a new pacing system in a remote location. An additional 10 to 14 days of intravenous antibiotics were given, followed by 1 week of oral antibiotics. No additional late complications or deaths occurred among any of the patients with a superficial or deep-pocket infection. $S$ epidermidis bacteremia without an identifiable source was observed in a 13-year-old patient with tetralogy of Fallot 3.5 years after implantation of a dual-chamber epicardial pacemaker. The patient was treated with vancomycin and netilmicin for 6 weeks, with resolution of the bacteremia. No subsequent pacemaker infection ensued.

\section{Predictors of Pacemaker Infections}

The 1- and 2-year freedoms from pacemaker (superficial and deep) infection were $97 \%$ and $93 \%$, respectively (Figure 1). No significant difference was observed in the incidence of superficial infections on the basis of a primary implantation. However, of the 9 deep infections, 8 were a 
revision of a previous lead, generator, or both. Only one patient had a deep-pocket infection after a primary pacemaker implantation, although this patient had undergone palliative cardiac surgery 3 weeks earlier. In total, those undergoing a second or subsequent pacemaker replacement were 2.5 times as likely to have a pacemaker infection compared with those undergoing a primary implantation ( $14.0 \%$ vs $5.6 \%$; relative risk [RR], 2.5; $P<.01)$. No additional risk of infection was observed on the basis of the era of implant in either the univariate $(P=.29)$ or multivariate analyses.

Of the 31 generators implanted in the 23 patients with trisomy $21,7(22.6 \%)$ patients had a superficial cellulitus, and $2(6.4 \%)$ had a deep-pocket infection requiring pacemaker removal. Patients with trisomy 21 were thus nearly 4 times as likely to have a pacemaker infection compared with the remainder of the cohort (25.8\% vs $6.5 \%$; RR, 3.9; $P<$ $.01)$. In univariate analyses single-chamber pacemakers had a higher incidence of infection than dual-chamber systems (single chamber: $10.2 \%$ vs dual chamber: $4.3 \%$; RR, $0.4 ; P<$ .05). However, this association is confounded by the high rate of single-chamber pacer use among patients with Down syndrome, who were more likely to experience pacer infections. Age or weight at implantation, presence of congenital heart disease, implantation during a concomitant cardiac procedure, or era of implantation did not predict the development of a superficial or deep pacemaker infection. Although no difference in the incidence of superficial infections was identified with the position of the pacemaker pocket, all 9 deep-pocket infections occurred with a subcutaneous or preperitoneal pocket $(P<.01)$. No deep pacemaker infection occurred in this series when a subpectoral pocket was used. Use of temporary epicardial or endocardial wires before permanent pacemaker implantation did not alter the risk of infection. The use of perioperative antibiotics could not be assessed as a prophylactic measure in the prevention of pacemaker infections because greater than $90 \%$ of the cohort received antibiotics for at least 24 hours after the operation.

\section{Discussion}

Permanent pacemakers have had a considerable effect in the management of children with atrioventricular block, symptomatic sinus node dysfunction, and, recently, as an adjunct in the therapy of tachyarrhythmias. 1,2,7-10 Although advances in technology and improving expertise in lead implantation have made pacemaker implantation relatively simple and safe, caution must be exercised when implantation of a foreign body occurs.

Because most of the experience with pediatric pacemaker infections results from anecdotal cases, we sought to report the incidence, possible predictors, and management strategies involving pacemaker infections in children over the last 20 years. Furthermore, because most of the adult experience involves transvenous leads, little information has been reported with epicardial pacemaker infections.
Early infections after pacemaker implantation are thought to result from wound contamination at the operation. ${ }^{5}$ Most of these infections appear to manifest within the first 2 months after the operation and are isolated within the generator pocket. ${ }^{3,5}$ On the basis of criteria by Choo and associates, ${ }^{6}$ we identified an early incidence of superficial infection of $4.4 \%(n=17)$ and deep-pocket infection of $1.5 \%$ $(n=6)$. Although the incidence of deep-pocket infections in our study is similar to incidences seen in large adult cohorts (0.7\%-1.0\%), our incidence of superficial infections is greater than the $0.8 \%$ reported in adult studies. ${ }^{3,4}$ However, in both our pediatric population and the comparative adult studies, all superficial infections were treated successfully with antibiotics and did not require additional operations.

Surgical infections occur when the combination of microbial numbers and virulence is sufficiently large enough to overcome the local host defense mechanisms. Wound infections tend to be more common in the young ${ }^{11}$ and might relate to immature resistance mechanisms. Although strict adherence to sterile surgical technique has dramatically reduced the incidence of surgical infections, there continue to be wound infections partly related to breaks in technique but also caused by reduced host resistance. In our study, patients with Down syndrome were 4 times as likely to have a pacemaker infection than the remainder of the cohort. Abnormalities in humoral and cell-mediated immunity have been described in patients with Down syndrome with reduced levels of immunoglobulin $\mathrm{M}$, zinc, total lymphocyte counts, $\mathrm{T}$ and $\mathrm{B}$ lymphocytes, and $\mathrm{T}$ helper and suppressor cells. ${ }^{12}$ In a prospective study Licastro and colleagues ${ }^{13}$ found that 4 months of oral zinc supplementation in patients with Down syndrome resulted in normalization of thymulin, increased lymphocyte proliferation and polymorphonuclear activity, with a significantly reduced incidence of infections. Alternatively, prophylactic use of vancomycin in high-risk patients (ie, patients with Down syndrome) might reduce the incidence of superficial infections.

The time interval between pacemaker implantation and infectious symptoms is often quite variable. Although superficial pocket infections tend to occur early in the adult experience, deep-pocket infections involving the electrode tend to occur later (median, 7-8 months). ${ }^{14} S$ aureus and epidermidis appear to be the most consistent organisms identified from adults with pacemaker-mediated infections. Staphylococcus species was identified in $44 \%$ of the deep pacemaker infections in our cohort.

Appropriate management of pacemaker-related infection involves the complete removal of the generator and leads, regardless of the extent and location of the infection. Delay or failure to remove the entire pacemaker apparatus (leads and generator) with a deep-pocket infection has been reported to result in a mortality of $25 \%$ to $66 \%$ in adult studies. ${ }^{15,16}$ Conservative therapy with systemic antibiotics as the mainstay 
of therapy without removal of foreign material has also been reported to fail in $97 \%$ of patients. ${ }^{6}$ The 2 patients in our cohort who underwent partial lead extraction had a recurrent infection necessitating a further operation to remove the remaining lead. Our current approach and recommendations for deep pacemaker infections involves intravenous antibiotics, removal of all foreign material, and reimplantation at a remote site during a single operation. The current availability of laser lead extraction might aid in the removal of all infected transvenous hardware.

Use of a temporary pacing wire and repeat operations for noninfected complications (principally lead revision) have been identified as risk factors in pacemaker-mediated infections. ${ }^{4,17,18}$ Although temporary pacing leads were only used in $6.8 \%$ of endocardial leads and $21 \%$ of epicardial leads, no increased risk could be identified in our patient population. It has been suggested that the adults who require temporary transvenous pacing are generally sicker and have been identified preoperatively as being at high risk (ie, with diabetes mellitus, leukemia, malnutrition, or long-term steroid use). ${ }^{17}$ However, similar to the adult studies, of the 9 patients who had a deep-pocket infection in our cohort, all but one occurred as a revision of a prior pacing system. These indications include a lead change for fracture, poor pacingsensing, or an isolated generator change. Although it seems logical that reopening the wound might predispose to infection, the exact pathophysiologic mechanism is less well understood. The pacemaker pocket in a reoperated system might be less well vascularized and have a decrease in tissue oxygen tension, resulting in an increased susceptibility to bacterial contamination, despite prophylactic antibiotics. In addition, although pediatric pacemaker implantation has evolved from a predominantly epicardial to endocardial approach, one of the potential problems is the very thin subcutaneous tissue, which often leaves the child with an unpleasant cosmetic result and renders the incision more susceptible to erosion and infection. Our approach over the last 5 years has been to place all endocardial pacemakers under the pectoralis major muscle. The absence of a deeppocket infection in any of the 82 pacemakers placed through a subpectoral incision is consistent with earlier results by Gillette and coworkers, ${ }^{19}$ who found the subpectoral pocket to be the preferred site.

This study is limited by its retrospective data collection. Furthermore, it is unknown to what extent antibiotics prevented any of the superficial infections from progressing to a deep-pocket infection.

In conclusion, superficial and pacemaker deep-pocket infections occur less than $5 \%$ of the time, with Down syndrome and reoperations accounting for the majority of infections. Primary pacemaker implantation carries an exceedingly low risk of deep-pocket infection. As evidenced by this study, the management of any deep-pocket infection should include removal of the generator and leads in their entirety. In the 2 instances of deep pacemaker infection whereby only partial transection of the epicardial leads was performed, both patients returned with a recurrent infection. Further prospective work identifying and treating high-risk patients might result in a lower incidence of wound infections and a need for generator and lead extractions.

\section{References}

1. Cohen MI, Bush DM, Vetter VL, Tanel RE, Wieand TS, Gaynor JW, et al. Permanent epicardial pacing in pediatric patients: 17 years of experience and 1,200 outpatient visits. Circulation. 2001;103:258590.

2. Beaufort-Krol GCM, Mulder H, Nagelkerle D, Waterbolk TW, BinkBoelkens MT. Comparison of longevity, pacing, and sensing characteristics of steroid-eluting epicardial versus conventional endocardial pacing leads in children. J Thorac Cardiovasc Surg. 1999;117:523-8.

3. Kiviniemi MS, Pirnes MA, Eranen HJK, Kettunen RVJ, Hartikainen JEK. Complications related to permanent pacemaker therapy. Pacing Clin Electrophysiol. 1999;22:711-20.

4. Aggarwal RK, Connelly DT, Ray SG, Ball J, Charles RG. Early complications of permanent pacemaker implantation: no difference between dual and single chamber systems. Br Heart J. 1995;73:571-5.

5. Bluhm GL. Pacemaker infections- A 2-year follow-up of antibiotic prophylaxis. Scand J Thorac Cardiovasc Surg. 1985;19:231-5.

6. Choo MH, Holmes DR, Gersh BJ, Maloney JD, Merideth J, Pluth JR, et al. Permanent pacemaker infections: characterization and management. Am J Cardiol. 1981;48:559-64.

7. Hayes DL, Holmes DR, Maloney JD, Neubauer SA, Ritter DG, Danielson GK. Permanent endocardial pacing in pediatric patients. J Thorac Cardiovasc Surg. 1983;85:618-24.

8. Fishberger SB, Wernovsky G, Gentles TL. Long-term outcome in patients with pacemakers following the Fontan operation. Am J Cardiol. 1996;77:887-9.

9. Ramesh V, Gaynor JW, Shah MJ, Spray TL, Vetter VL, Rhodes LA. Comparison of left and right atrial epicardial pacing in patients with congenital heart disease. Ann Thorac Surg. 1999;68:2314-9.

10. Walsh CA, McAlister HF, Andrews CA, Steeg CN, Eisenberg R, Furman S. Pacemaker implantation in children: a 21-year experience. PACE. 1988;11:1940-4.

11. Dellinger EP. Surgical infections and choice of antibiotics. In: Sabiston D, editor, Textbook of surgery. 15th ed. Philadelphia: W.B. Saunders; 1997. p. 264-80.

12. Lockitch G, Singh VK, Puterman ML, Godolphin WJ, Sheps S, Tingle AJ, et al. Age-related changes in humoral and cell-mediated immunity in Down syndrome children living at home. Pediatr Res. 1987;22: 536-40.

13. Licastro F, Chiricolo M, Mocchegiani E, Fabris N, Zannoti M, Beltrandi E, et al. Oral zinc supplementation in Down's syndrome subjects decreased infections and normalized some humoral and cellular immune parameters. J Intellect Disabil Res. 1994;38:149-62.

14. Mezlis N, Hough RE, Oakley GDG. Infections in transvenous cardiac pacemakers: 2 more cases. PACE. 1997;20:2992-4.

15. Phibbs B, Marriott HJL. Complications of permanent transvenous pacing. N Engl J Med. 1985;312:1428-32.

16. Rettig G, Doenecke P, Sen S, Volkmer I, Bette L. Complications with retained transvenous pacemaker electrodes. Am Heart J. 1979;98:58794.

17. Mounsey JP, Griffith MJ, Tynan M, Gould FK, MacDermott AFN, Gold RG, et al. Antibiotic prophylaxis in permanent pacemaker implantation: a prospective randomized trial. Br Heart J. 1994;72:33943.

18. Chauhan A, Newell GSA, Stone NDL, Shapiro LM, Schofield PM, Petch MC. Early complications after dual chamber versus single chamber pacemaker implantation. PACE. 1994;17:2012-5.

19. Gillette PC, Edgerton J, Kratz J, Zeigler V. The subpectoral pocket: the preferred implant site for pediatric pacemakers. PACE. 1991;14: 1089-92. 